\title{
Culturally adapted Family Intervention (CaFI): case examples from therapists' perspectives
}

\author{
Katherine Berry*,1, Christine Day ${ }^{2}$, Lee D. Mulligan², Tara Seed ${ }^{2}$, Amy Degnan ${ }^{1}$ and \\ Dawn Edge ${ }^{1}$
}

${ }^{1}$ Division of Psychology and Mental Health, School of Health Sciences, Faculty of Biology, Medicine and Health, University of Manchester, Manchester Academic Health Science Centre, Manchester, UK ${ }^{2}$ Greater Manchester Mental Health NHS Foundation Trust, Manchester Academic Health Science Centre, Manchester, UK

Received 20 October 2017; Accepted 30 July 2018

\begin{abstract}
This paper describes three case examples from a recent trial of family intervention specifically designed for people of African-Caribbean descent. These examples, told from the therapists' perspectives, highlight key components of the intervention and issues that arose in working with this client group. Findings from the study suggest that it is possible to engage this client-group in family therapy similar to traditional evidenced-based family interventions, although as illustrated in the paper, it is important that therapists pay attention to themes that are likely to be particularly pertinent for this group, including experiences of discrimination and mistrust of services. The use of Family Support Members, consisting of members of the person's care team or volunteers recruited from the community, may also help support people to engage in therapy in the absence of biological relatives.
\end{abstract}

Key words: psychosis, family approaches, CBT, clinical trial

\section{Introduction}

Family intervention (FI) is clinically and cost-effective for psychosis (Pharoah et al., 2010; NICE, 2014). Research has consistently shown that undertaking FI with service users diagnosed with schizophrenia and their families improves outcomes (Lobban et al., 2013; Pharoah et al., 2010). For example, in their Cochrane Review, Pharaoh and colleagues (2010) reported that FI improved medication compliance, self-management and problem-solving, which were associated with reduced risk of psychotic relapse. In addition to decreasing or preventing relapse, improving service users' social functioning, and enhancing their

\footnotetext{
*Author for correspondence: Dr Katherine Berry, Division of Psychology and Mental Health, School of Health Sciences, Faculty of Biology, Medicine and Health, University of Manchester, Manchester Academic Health Science Centre, 2nd Floor, Zochonis Building, Brunswick Street, Manchester M13 9PL, UK (email: Katherine.Berry@ manchester.ac.uk).
} 
quality of life, a key aim of FI is to reduce carer burden and ill-health (Lobban et al., 2013).

Although there are a number of approaches to FI, they share common core components such as psycho-education, problem solving, stress and crisis management, and enabling carers to practise good self-care. Core principles of FI models include: a holistic approach to care and treatment, establishing therapeutic alliance, addressing family tension, and setting reasonable and achievable goals and expectations. The National Institute for Health and Care Excellence (NICE, 2014) recommends at least 10 sessions of FI for people with schizophrenia diagnoses who are in contact with their families.

FI might particularly benefit people from African-Caribbean backgrounds who have the highest incidence of psychosis in the UK (Qassem et al., 2015; Tortelli et al., 2015). Long duration of untreated psychosis (DUP) increases family stress and carer burden (Keating and Robertson, 2004). However, FI is rarely available in the National Health Service (NHS). Haddock and colleagues (2014) audited the files and electronic notes of 187 randomly selected individuals with a diagnosis of schizophrenia and found that implementation rates for FI were $1.6 \%$ 'offered' and $1.1 \%$ 'delivered'. Unavailability of FI might disproportionately affect black and minority ethnic (BME) service users in general as they are known to have inferior access to psychological therapies (Morgan et al., 2004). African-Caribbean service users are likely to have even poorer access to FI due to the high levels of family disruption (Keating and Robertson, 2004). NICE guidance for schizophrenia (NICE, 2014) highlights the needs to culturally adapt and evaluate psychological interventions for ethnic groups to improve access. Our own consultations with local African-Caribbean community mental health groups also found dissatisfaction with the lack of access to psychological therapy and expressed a strong desire for culturally appropriate 'talking treatments'. In addition, people wanted to develop better understanding of schizophrenia and improve relationships within families and between African-Caribbean communities and with mental health services.

In Edge et al. (2016) we describe a protocol for a study to culturally adapt the Barrowclough and Tarrier (1992) model of FI specifically for people of African-Caribbean descent and to assess its acceptability and feasibility in an open trial with 30 family units. The Culturallyadapted Family Intervention (CaFI) is delivered over 10 sessions within a 20-week therapy window and is focused on five key phases: assessment and engagement; shared learning; communication; problem-solving, coping and goals setting; and planning for the future including relapse prevention. A novel aspect of CaFI therapy is the use of family support members (FSMs), enabling involvement of service users without access to biological family members either due to mistrust of services or breakdown in family relationships; factors that are particularly pertinent within this client group (Keating and Robertson, 2004). FSMs were either community volunteers recruited by the study team or significant others nominated by service users, including friends or mental health professionals who were already involved in participants' care, for example key nurses or care co-ordinators. Social networks often diminish in psychosis, with mental health staff often playing key roles due to breakdown in family or peer relationships (Berry et al., 2007). Communication and other relationship difficulties between staff and service users can therefore be particularly pertinent and would benefit from intervention (Berry et al., 2011).

The CaFI study was approved by North West Greater Manchester East National Research Ethics Service (NRES) Ethics Committee (13/NW/0571). The feasibility trial found that it 
was feasible to recruit participants and either family members or FSMs to the trial. Once recruited, there were good levels of engagement and retention within therapy sessions, with $77 \%$ of families completing therapy and $92 \%$ attending all ten sessions offered (Edge et al., 2018). Quantitative and qualitative evaluation further suggested that the intervention was acceptable to key stakeholders (Edge et al., 2018). However, as a feasibility pilot, the study was not powered to estimate changes in health outcome, and did not include a control group as comparison.

Here we present three case examples from the trial as seen from the therapists' perspectives which provides a richer description of the intervention described in the main outcome paper from the trial and the therapy manual (available upon request). We have selected these particular cases as they provide examples of key compositions of 'family units' and settings that we experienced within the trial. The first case ('Samuel') is young man living at home with his mother and as such represents the typical mother-son dyad seen in family work with people with psychosis. The second case is a man ('John') residing within a psychiatric intensive care unit (PICU) mental health setting who was supported by a FSM from the community. The third case is a woman ('Sharon') living in a supported tenancy in the community supported by her care co-ordinator.

Three separate pairs of therapists, consisting of a 'lead' and 'co-therapist', delivered the intervention. All lead therapists were clinical psychologists and met the British Association for Behavioural and Cognitive Psychotherapy eligibility criteria for accreditation as cognitive behavioural therapists, with one therapist also training in cognitive analytic therapy. Two of the lead therapists had experience in working with people with psychosis and their families within community mental health teams, whilst the other lead therapist was more experienced in child and family work. Co-therapists had experience of working as support workers or nurses with people with psychosis but were not training or experienced in therapy delivery. Although all of the therapists had had previous experience in working with ethnic minority groups including people of African-Caribbean descent, none described themselves as being specialists in delivering culturally adapted therapies. In addition to training and supervision in the manual delivered by the lead author, all therapists also underwent additional cultural competency training. This was delivered by Just Psychology, a Manchester-based Community Interest Company, specializing in the delivery of culturally sensitive training and psychological therapy. We also commissioned Meriden Family Programme to provide additional training for the lead therapists in 'Family Interventions and Cultural Competence'. Key components of the training delivered by Just Psychology and Meriden Family Programme included:

- Core competences to work effectively with families experiencing schizophrenia and psychosis;

- Current legislation and how it relates to clinical practice (such as The Equality Act 2010, Protected Characteristics, NHS Knowledge Skills Framework and Public Sector Equality Duty);

- Cultural awareness and family work practice (for example, the potential impact of culture and family experience on CaFI's delivery);

- Relationship between racism, discrimination, adversity and mental illness;

- Effect of power and prejudice in building trusting therapeutic relationships. 


\section{Case studies}

Each participant and their family members or FSMs have been given a fictitious name for the purposes of this report to maintain anonymity. Each case study will be presented by providing contextual information to set the scene, followed by therapist reflections on each stage of the therapy including any observations or key learning points to demonstrate the approach in practice. All cases described followed the same session order: assessment/engagement, shared learning, communication, coping skills and endings. In some instances we varied this order based on the formulation, e.g. communication was addressed first due to the significant impact of communication difficulties during the sessions, but not in the cases described.

\section{Samuel}

\section{Context}

Eighteen-year-old 'Samuel' was of 'mixed' heritage. His father originated from the Caribbean and his mother, Michelle, whom he lived with, was White British. At the time of the study, Samuel was accessing support from an early intervention for psychosis service (EIPS) following a first episode of psychosis (FEP). Samuel's symptoms included auditory hallucinations, suspiciousness and persecutory beliefs. He also had a diagnosis of attention deficit disorder (ADD) and an auditory processing disorder. Samuel was prescribed risperidone (anti-psychotic medication), which he did not take during the majority of the intervention period of the trial.

Samuel and Michelle both recognized that, despite having a good relationship, arguments between them had increased and often resulted in Michelle calling the police. Michelle described Samuel as aggressive and bullying, and Samuel felt that Michelle 'nagged' and criticized him.

Samuel and Michelle attended ten sessions of therapy over a 4-month period. All sessions were delivered in the family home. Therapy was initially started with a pair of male therapists, but following the first session, Samuel asked if he could see female therapists instead as he did not feel comfortable working with men. Although Samuel and Michelle were the only family members who lived together and took part in the therapy, relationships with other family members were also discussed as part of the therapy. This included: Samuel's older sister Kate, who had a diagnosis of schizophrenia and lived in supported housing; his father, Michael, whom he saw every few weeks and who had separated from Michelle during Samuel's childhood; and Michelle's new partner Stuart, who been in a relationship with Michelle for several years and lived in the family home. Unfortunately, neither Michael nor Kate wished to participate when they were approached about the study. Similarly, although Michelle's partner, Stuart, lived with Michelle and Samuel, he experienced difficulties in attending sessions regularly due to work commitments.

\section{Engagement}

Initially during therapy, Michelle dominated the conversation and expressed her frustration that Samuel did not take his medication. She described 'going through this process before' with Samuel's sister, Kate, who had a diagnosis of schizophrenia. Michelle remained confident that if Samuel would take his medication, this would resolve the arguments they 
had and reduce Samuel's aggression. Samuel did contribute when asked about his perspective but his responses were often brief or he was interrupted by Michelle. The conversation was often loud and evolved into shouting, with Michelle becoming angry about Samuel not taking his medication or following house 'rules'. Despite these arguments, prior to and throughout therapy, they both talked about having conversations with each other every evening and could identify positives in their relationship. Samuel and Michelle's primary shared goal was to improve the way in which they communicated and this was operationalized as a SMARTER (Specific, Measured, Achievable, Realistic, Timed Evaluated and Rewarded) goal: specifically, the length of time they spent together in mutually rewarding conversations about each other's day and a reduction in the number and severity of arguments that they had each week.

\section{Shared learning}

Understanding Samuel and Michelle's views about psychosis and sharing information about the impact of stress on wellbeing and symptoms related to psychosis was an important part of therapy. Samuel felt that people who had not experienced psychosis could not understand it. He shared some of his experiences during the therapy session and Michelle often reflected she had not known some of the things that he talked about, for example feeling that people at college were talking about him and experiencing very threatening and distressing voices. In addition, Samuel reported experiencing people at college making derogatory and threatening comments about his race, although it was not always clear if these reflected threats were made by actual people or were a voice-hearing experience. Michelle also used space within sessions to discuss her own upbringing and how experiences of abuse and neglect during her own childhood might have led her to flip between feeling threatened by Samuel's aggression and feeling like she wanted to protect him.

\section{Communication}

Although communication was a theme that was addressed throughout therapy, several sessions specifically aimed to reduce arguments between Samuel and Michelle. For example, therapy focused on trying to understand episodes of conflict by drawing out mini-formulations, taking into account each person's perspective and depicting how things could quickly escalate through lack of communication and consequent misunderstanding of each other's viewpoints or motives.

Samuel and Michelle practised expressing positive and negative feelings within the sessions and as a between-session task. Using the communication sheets (available on request) outlining skills in active listening and tips about how to express positive and negative feelings (available upon request) allowed Michelle and Samuel to experiment with talking to each other in different ways when discussing recent events and this appeared to lead to improved communication within the therapy sessions.

\section{Coping skills}

Sessions on coping skills and problem solving for Samuel and Michelle provided a further opportunity to address communication skills which seemed to be particularly problematic for this family. Samuel found it difficult to express his feelings and at times he had not felt confident that others would help him. However, as therapy progressed he started to 
communicate more readily with his care coordinator and people at college when he needed support. Michelle recognized her own needs for self-care and started to engage more often with activities outside of the home. Both Michelle and Samuel practised problem solving in relation to disagreements within the home. For example, Samuel talked loudly on his mobile 'phone at night which often kept Michelle awake, so they were able to reach a compromise whereby he would not talk to people on the 'phone in the house after a certain time during the evenings.

\section{Ending and reflections}

The therapists were initially concerned that the family would disengage with them given that Samuel had not been able to engage with the male therapists. The therapists queried whether Samuel genuinely had an issue with the previous therapists' gender or was looking for reasons to not engage in what was potentially a difficult process as evidenced by his relatively less vocal contribution to initial sessions. The therapists were White British and expressed concerns in supervision about whether this would make it hard to empathize with Samuel's experience of racism and that they might unintentionally express views that Samuel might misconstrue as being racist. The latter was a particular concern as Samuel was vulnerable to experiencing paranoia.

However, both Michelle and Samuel reported finding the intervention helpful and Samuel engaged well and became increasingly more vocal throughout the intervention. Throughout therapy, Michelle often expressed that she would have wanted the intervention to continue for a longer period of time. It is also noteworthy that Samuel's care co-ordinator reported that she noticed an improvement in Samuel's confidence and the high levels of distress, as well as the concern Michelle vocalized to services in relation to her son's well-being.

Samuel's father, Michael, and sister, Kate, were often referred to during sessions and it was hypothesized that they both influenced Samuel and Michelle's relationship, as well as Samuels's limited sense of achievement and poor self-esteem. On reflection, it would have been beneficial to engage them in the therapy to help imbed wider systemic change and to maintain the progress Samuel and Michelle had achieved within therapy.

\section{John}

\section{Context}

John was a 34-year-old single gentleman who was born in the UK but whose family had originated from the Caribbean. During the therapy, he resided in a ten-bedded all male PICU. He had been admitted to the PICU from an adjoining ward due to repeated absconsions and frequent use of illicit substances. He was admitted under Section 3 of the Mental Health Act (MHA). John had been first diagnosed with schizophrenia 7 years previously following involuntary admission to an acute psychiatric ward. Since then, he had experienced numerous inpatient admissions under sections of the MHA often involving the police to bring him into hospital.

John was prescribed clozapine (anti-psychotic medication) and utilized diazepam as required throughout the therapy period. However, following a period of absconding when John stopped taking his medication, his clozapine was re-initiated and gradually titrated again to the highest tolerable dose. This led to a 3-week delay in therapy, in line with John's request, 
as his mood deteriorated significantly. John attended therapy over a period of 20 weeks, during which he attended the maximum number of 10 sessions. As John identified no family members in the local area, six sessions were attended by a FSM, April, whose role was to support John, advocate for him, and facilitate the completion of therapy and homework tasks. The FSM was an African-Caribbean, female volunteer recruited from the community who had previously trained as a social worker.

\section{Engagement}

During the engagement phase of therapy, John described having a number of traumatic experiences in childhood and adulthood and referred to the relationship with his biological family as 'distant'. John's formulation made links between alleged experiences of childhood sexual abuse, a history of traumatic loss (of close friends who were killed or unintentionally overdosed in his presence), and experiences of racial discrimination (being treated unfairly by the police and perceiving apathy from health professionals involved in his care), in the development of his core beliefs (about himself being vulnerable and worthless, others being abusive, and the world being unfair and dangerous). John had a history of coping with difficulties by using illicit substances, most commonly cocaine and cannabis. He also avoided difficult situations by withdrawing from others.

John identified several problems he hoped therapy could address. These included anxiety, auditory hallucinations, low mood and worry. John also identified three goals for therapy, which were operationalized into SMART goals. Collaborative plans were also made to ensure all goals were consistently reviewed and monitored for progress.

John's goals included:

(1) To feel more relaxed: operationalized by a reduction in the frequency of panic attacks, the development of coping strategies for anxiety and a reduction in auditory hallucinations (specifically, experiences of 'sound enhancement').

(2) To feel better in mood: identified by an improvement in self-reported mood (rated 4/10 at the beginning of therapy), and increased involvement in meaningful activities (i.e. listening to, and creating, music).

(3) To spend less time ruminating on the past or worrying about the future: identified by a reduction in the time spent engaging in unhelpful extended processing (estimated to be approximately 4 hours each day at the beginning of therapy).

The goals of April were to learn more about the symptoms of psychosis and the mental health services from a first-hand perspective and to support John in achieving his therapy goals.

\section{Shared learning}

During the early phases of therapy, it became clear that John held a culturally specific explanatory model of his mental health difficulties. In particular, he believed his experiences were caused by his father, who John believed was 'controlling' his actions and emotions, via the mechanism of voodoo. He also reported that no professional had ever explained the meaning of his diagnosis to him. Therefore, John was provided with psychoeducational materials to help him understand the label of 'schizophrenia', normalize his difficulties, and socialize him to the link between trauma, illicit drug use and mental health problems. 


\section{Coping skills and communication}

Given the external attribution John had about his mental health difficulties, mood monitoring was introduced to help him recognize the link between thoughts, feelings and behaviours and to increase his sense of agency over his experiences. Mood diaries were implemented, and following this, behavioural activation was utilized to increase John's activity and to facilitate his goal of addressing low mood. This also helped John to reduce what he referred to as 'idle time', in which he would worry about the future or dwell on the past.

John rated his anxiety as severe on rating scales and he experienced regular panic attacks. However, he had very few coping strategies other than taking diazepam, withdrawing from others, or 'curling into a ball'. During therapy, John struggled to work at a cognitive level, especially when discussing his experience of anxiety. Therefore, a behavioural intervention, progressive muscle relaxation, was introduced to help John regulate his feelings of anxiety. Mood monitoring also helped John to recognize the early signs of anxiety, which cued him to implement relaxation strategies. Notably, John could recognize how staff could support him during times he was experiencing anxiety, but struggled to communicate his feelings to them. Therefore, communication skills were introduced to help John practise these skills, and build his confidence in communicating his needs to his care team.

\section{Ending and reflections}

Prior to commencing therapy, the therapists were reticent about working within an inpatient setting where typically patients have limited access to therapy and are sometimes considered too unwell by ward staff to engage in regular therapy sessions. However, despite these initial reservations, the therapists were able to adapt to the inpatient environment by offering John breaks during therapy when he was struggling with his concentration and communicating regularly with ward staff so they were aware of the work that was being carried out and dispel any myths. Overall, John made significant improvements during therapy. John's self-rated mood increased from 4/10 to 8/10 and he reported a noticeable reduction in the time he spent engaging in extended processing (reporting approximately 30 minutes each day compared with 4 hours per day at the beginning of therapy). These improvements were acknowledged by his care team, who described John as 'more positive' on the ward. John also described feeling better able to manage his anxiety through implementing relaxation strategies and seeking support from ward staff.

Improved communication skills helped John to engage more effectively, and both he and staff reported a significant improvement in their relationship. John was able to express his wishes to staff and requested they reduced the clinical information they shared with his father was controlling his actions or emotions against his will.

Shortly following therapy, John was transferred to a low-secure rehabilitation ward to facilitate future transfer into community services. John was able to identify future recovery goals, which included playing music in public, being more independent with respect to his mental health and further reducing his anxiety.

Throughout the course of therapy, April (FSM) facilitated a number of therapeutic tasks. By reflecting on her personal experiences, April helped to normalize and validate John's experience of anxiety and reinforce the helpful strategies (namely, relaxation and seeking support from staff) that were introduced. April also shared personal stories about her own family, which helped to develop a shared cultural narrative between her and John. However, 
inevitably, the inclusion of a FSM also created some barriers. At times, April's own anxiety about the therapy and the PICU setting impeded her ability or willingness to engage in role plays or support John with homework. This highlighted the importance of training and supervision to ensure FSMs feel confident, willing and able to support interventions delivered in inpatient settings.

\section{Sharon \\ Context}

Sharon was a 45-year old, single woman who had been residing in supported accommodation in the community for many months pending a move to accommodation that supported more independent living. Sharon had a longstanding diagnosis of paranoid schizophrenia and a more recent co-morbid diagnosis of depression. Sharon had been admitted as an inpatient on many occasions since being a young adult. Sharon reported that she had never engaged in any therapeutic intervention prior the study and she was initially ambivalent towards the process. She described how she was previously offered therapy but had been discouraged to take it up by members of her family who felt that talking to strangers about her problems would not help. She had been prescribed and was taking clozapine (anti-psychotic medication) and anti-depressant medications as part of her overall treatment.

Prior to setting up the therapy, Sharon reported having a close relationship with her brother, despite sometimes having arguments with him. However, she felt that he could not participate in the trial due to his own low mood and poor mental health. Sharon did not think that her father would engage with the trial and did not want him to be approached about the study. Sharon felt that he had other commitments with his family and other children and did not like to talk openly about family problems. Sharon wanted to build a relationship with her care-coordinator Jackie and therefore invited her to participate in the trial. Jackie was an experienced community mental health nurse who had known Sharon for approximately one year when she had been assigned to Sharon following a reorganization of care teams. Prior to commencing therapy, Sharon and Jackie typically met fortnightly to review Sharon's mental health. Jackie was White British and had limited knowledge of African-Caribbean culture but had limited her queries about it in her work with Sharon due to a fear of appearing racist.

Sharon engaged in a total of 10 sessions over a 3-month period. The sessions took place on the same day, weekly and at the same time. This consistency was to ensure that Sharon would remember the sessions and it helped ensure predictability and regularity, which would support engagement and trust in the therapeutic alliance. The side-effects of her medication often left her feeling drowsy in the mornings, therefore sessions were planned for a time when she was more awake. Sharon set herself two goals. Firstly, she wanted to understand why she had developed mental health problems and what was keeping her symptoms going and secondly, she wanted to develop ways of coping with her symptoms. Jackie's goal was also learning more about Sharon and improving her capacity to support Sharon in managing her symptoms.

\section{Engagement}

During the assessment and engagement phase of therapy, Sharon described a number of traumatic earlier experiences. She described her relationship with her parents as critical, 
rejecting and confusing. Sharon experienced being the child who was pitied by the wider family unit. Sharon alleged earlier sexual abuse which left her feeling bad about herself and mistreated by others. During adolescence, Sharon described how she 'went off the rails' which consisted of forming inappropriate sexual relationships with many men and illicit drug use.

Around this time, she experienced the sudden and tragic loss of a close friend and her grandmother, who the formulation suggested was her secure attachment figure. This was the onset of what she described as being her initial psychotic episode. She experienced paranoia, low mood and hearing voices. Throughout her earlier adulthood she had been admitted to several inpatient units and she described how she experienced control, mistreatment and emotional neglect in the care of people with a similar ethnic background. She described how mental illness within her culture and her family unit was believed to be bad and brought shame to the family. This influenced how she began to see herself and left her feeling shunned and excluded from the family. During therapy, Sharon explored how her key earlier experiences had influenced the beliefs she had formed about herself ('I am bad, unlovable, disgusting, ugly'), others ('other people can't be trusted, are abusive and controlling') and the world as an unsafe, threatening place ('I will be attacked' 'bad things happen in life'). These beliefs had a profound impact on how she related to others. Sharon's mistrust in others and paranoia meant that she believed that people were talking about her, and that people were planning to hurt her. These cognitive misinterpretations exacerbated her paranoia. She would cope by withdrawing from meaningful relationships or responded to people with mistrust, which in turn would have a negative impact on her relationships and leave her feeling alone and isolated. These thoughts and coping responses had a significant impact on the relationship with her care co-ordinator and the support staff in her accommodation. Sharon would often respond with agitation and verbal aggression when she felt controlled or when she felt she was instructed to do something. Sharon would also withdraw and isolate herself in her bedroom when she felt that staff were too involved in her day-to-day activities. The staff from Sharon's supported tenancy had previously reported to Jackie that they experienced Sharon's mood as being unstable.

Despite the length of time that they had known each other, Jackie knew little about Sharon's history so hearing about her past and the impact it currently had on her relationships, helped to improve Jackie's understanding of Sharon's needs and vulnerabilities. Within sessions Jackie was also able to provide reassurance and counter evidence to challenge Sharon's paranoid thoughts.

\section{Shared learning}

Sharon held a medicalized view of schizophrenia, meaning that she saw her symptoms as being caused by innate faulty brain chemistry and that medication should therefore be the main form of treatment. As indicated above, Jackie also had not had the opportunity to understand Sharon's experiences in the context of her life experiences. Therapy therefore helped Sharon and Jackie to understand the impact of stress and vulnerability factors on mental health, through the analogy of a stress bucket which can only take so much water (or stress) before it overflows. Therapy focused on exploring some of the labels around mental illness and schizophrenia to help understand their meaning and refute any negative connotations. The impact of stress and on symptoms such as voice-hearing and paranoia was discussed, and 
links were made between the nature of Sharon's past experiences (particularly sexual abuse) and current mistrust of others and her beliefs about and ways of relating to voices.

\section{Communication}

Sharon had numerous arguments with the staff in her accommodation and on occasion with her care coordinator, Jackie. Therapy focused on formulating what was causing and maintaining these arguments and Sharon noticed the pattern of agitation, withdrawal and shouting described above. Sharon and Jackie were asked to think through the benefits and the barriers to each of the communication skills: assertiveness, expressing positive feelings and expressing negative feelings. These skills were demonstrated in the session, practising each of them using hypothetical scenarios. Sharon practised with staff through between-session tasks with Jackie and the outcome was reviewed in the following session. A key moment of change for Sharon was being able to express her gratitude to staff whilst also communicating that she did not wish to do something or she did not wish for company. These key assertive skills enabled her to take control in a respectful way and it helped her develop closer relationships with staff.

Sharon reported that she wanted to be able to communicate assertively with her father. She often felt criticized and not good enough after she spoke with him. This had a significant impact on her mood and how she communicated with staff and the public. She described feeling neglected by him as he had another family and he would not visit. Sharon used the session to plan how she could assertively and respectfully express her feelings and needs to him. She practised with the support of Jackie between the sessions and used this to communicate more effectively with her father.

\section{Coping skills}

Sharon recognized her helpful and unhelpful coping strategies and therapy was used to map out how some strategies may have been helpful in the short term (relief of emotional suffering or protection), but unhelpful in the long term. The links between her thoughts, feelings and behaviours were drawn out during sessions and Sharon was able to recognize how her thinking reflected biases based on her past experiences rather than being an accurate reflection of the here and now. Within therapy, the themes of her thought biases were drawn out and alternative, more compassionate ways of thinking about herself were collaboratively generated with support from both the therapists and Jackie. Strengths of her positive voices and the inaccuracy of her critical voices were also explored. Sharon learned to 'act opposite' to the critical voice which gave it less power and control over her. For instance, remaining engaged and exploring her history within therapy was one example of how she acted against the voice which was instructing her not to talk about her past. Visual imagery was used in the sessions to support her to develop more compassionate images of people or voices that might help her, such as images of her late grandmother. At the behavioural level, progressive muscle relaxation was also introduced to help her manage her feelings of anxiety and paranoia and grounding techniques.

\section{Ending and reflections}

Sharon had a long history of mental health difficulties and experienced significant powerlessness and a lack of control in her relationship with others. These negative experiences were in the context of her family relationships and mental health services. Understandably, 
she found it hard to trust the therapists and her voices, which often criticized and denigrated her, were a barrier in the therapy sessions. The therapists highlighted these potential barriers early on in therapy as they sensed that the engagement process was not straightforward. As such they were able to formulate the barriers and use supervision to discuss how they might be overcome; for example, openly acknowledging Sharon's difficulty in trusting others and pinpointing instances when this might be impacting on the therapy relationship. Despite these barriers to therapy, Sharon continued with therapy and staff reported a significant change in Sharon's mood, evidenced by the fact that she was smiling more, communicating more effectively with them and getting along better with other residents in the accommodation. Sharon also reported having more understanding about her mental health difficulties and she reported feeling better in mood. She reported being extremely moved by her 'goodbye letter' (a letter which the therapists wrote at the end of the therapy to summarize the work carried out during the sessions, in addition to the person's strengths and development). Upon reflection, it might have been beneficial if her father and brother were involved with the therapy to support wider systemic change. However, as Sharon herself was reluctant for them to be involved in the study, it was not possible for the research team or the therapists to approach them directly.

\section{Discussion}

The above case examples highlight that despite the challenges, it was possible to engage a diverse range of participants both with and without biological family members in FI for psychosis. A number of key themes have been illustrated throughout the above case examples, some of which have been described previously in the context of therapy for psychosis (Fowler et al., 1995; Hagen, 2010) and others of which are more unique to this client group.

First, the potential role of earlier adverse experiences in the development of psychosis is illustrated by two of the cases (John and Sharon). It is now well established that earlier experiences of abuse, neglect, social exclusion or discrimination can play a causal role in psychosis (Bebbington et al., 2011; Varese et al., 2012). Although interpersonal traumas are over-represented in people with psychosis regardless of ethnic background, those from African-Caribbean groups may have been particularly likely to experience such adversities compared with their White peers (Morgan et al., 2007, 2017). The cases of John and Sharon also highlight how mental health services can repeat earlier patterns of abuse and negative treatment, thus fuelling negative perceptions of potential sources of support and future help seeking (Berry et al., 2013). It is well established that African-Caribbean people in particular may be more likely to receive coercive treatments within services including involuntary detentions and physical restraint (Schizophrenia Commission, 2012). The role of trauma in psychosis, and for this group of clients in particular, highlights the importance of exploring the client's psychosocial history within therapy, including their past experiences within services and helping them to make links between past experiences and their current beliefs about themselves or others and ways of coping with distress. The potential greater vulnerability to past loss experiences and negative relationships with services for this group of clients also means that ensuring a positive ending to therapy and smooth transition to any future care arrangements is especially important.

In contrast to the psychosocial model of psychosis, Michelle, Samuel's mother, had a medicalized view of mental health problems, believing that if Samuel just took his medication then his problems would be resolved. This belief represents a traditionally Western view 
of mental health problems and is notably in contrast to John's view that his mental health problems resulted from voodoo. Within therapy, Michelle was, however, able to reflect on the role of her own upbringing in determining the way she perceived her son's difficulties and interacted with him.

Second, the benefit of recruiting FSMs to the study was that people who had estranged or difficult relationships with their own family could still access a therapy which focused on family relationships and also benefit from support from significant others to engage in therapeutic tasks. For example, therapy with Sharon focused on helping her to communicate her feelings to her father even though he was not able or willing to engage in sessions, and Jackie helped to support Sharon in achieving this goal by practising communication skills both within and outside of sessions. An additional benefit of involving Jackie in therapy, as a care co-ordinator, was that Jackie could also learn more about Sharon's history and thoughts and feelings about past and present relationships, which served to strengthen their relationship. April was also able to support John, for example, by sharing her own anxiety and coping strategies which arguably could have helped to normalize difficult feelings that he was struggling with and help him to feel understood. Nonetheless, there were barriers to April's work with John which may have prevented her engaging with him more fully outside the sessions. In particular, April described how the secure nature of the setting John was in led her to feel uncomfortable about meeting with John outside of therapy to support him in completing between sessions tasks. The extent to which John and Sharon's cases are FI is also open to debate, which is why we wanted to present them in this paper. It could be argued that therapy for John and Sharon was more akin to culturally adapted CBT such as that described by Rathod et al. (2013). Although the therapies were not FI in the traditional sense, efforts were made in supervision to ensure that family relationships were discussed during therapy and supporting staff or volunteers were conceptualized as helping service users to develop healthy relationships which might extend to more positive relationships with biological family members in the future. In the context of some people's lives with psychosis, staff members also play significant roles in the absence of biological family, representing pseudo-family members (Berry et al., 2007).

Third, although the role of the FSM was valued in some respects, in the case of Sharon, the therapists did reflect that it might have been helpful to also engage Sharon's father and brother in therapy. Engaging her father in therapy may have been particularly important in the work on communication, as without his involvement there was no scope to explore with him how the way in which he interacted with Sharon might make her feel. The therapists also reflected that it may have been helpful if more members of Samuel and Michelle's family were involved in therapy as there were several instances when their relationships with Samuel had also had an impact on Samuel and Michelle's relationship and Samuel's self-identity. The reluctance for family members to engage in family therapy is a well-documented barrier to delivering FI (Berry and Haddock, 2008; Bucci et al., 2016). However, this is one barrier that may potentially be addressed with a longer period of engagement with therapy focused specifically on building relationships with significant others by trying to understand and break down the barriers leading to their reluctance. As suggested above, it is also possible that FSMs, particularly those who are part of the local community, may serve as a possible bridge between service users and biological relatives to help engage the latter into therapy.

Fourth, John's belief that his father was responsible for his symptoms via voodoo highlights a cultural specific belief about mental health problems. The therapy recognizes that people 
might have non-Western attributions for mental health problems and as such these are explored as part of shared learning. In fact we developed a tool as part of the study which explores beliefs about psychosis including beliefs about symptoms, causes and treatments that might be more common in African-Caribbean culture (Degnan et al., 2018). These culturally specific beliefs were based on a review of the literature and focus groups with AfricanCaribbean service users and their relatives. A key component of therapy involves therapists and families engaging in a process of discussing different beliefs and exploring the pros and cons of different ways of viewing situations and symptoms in a non-judgemental way.

Fifth, as alluded to above in the case of therapy with FSMs, reflections by the therapist and findings from our assessment of intervention fidelity (Edge et al., 2018) suggested that in some instance sessions were more akin to individual therapy as opposed to family therapy. In these instances, FSMs did not play a very active role in therapy and this was particularly evident when, due to participant risk issues or other commitments of FSMs, FSMs were not able to meet with participants outside of the therapy sessions. Further work needs to be undertaken to determine whether therapy with FSMs represents a different intervention. Nevertheless, it is important to note that half of the service users who started therapy in this study would not have been able to participate without FSMs, suggesting a role FSMs may have for other socially isolated groups.

Finally, the degree to which therapy focused on culturally specific issues varied throughout therapy and across cases, and it is noteworthy that during the initial phases of the research programme when we culturally adapted the traditional model of FI developed by Barrowclough and Tarrier (1992), service users and carers we consulted felt that all the content of the original manual was potentially relevant. However, those we consulted did feel that greater emphasis was needed upon communication than in the original model due to the higher levels of family breakdown within the group. Other key aspects of the cultural adaptation involved exploring and seeking to understand values and beliefs that were particularly pertinent to this client group, such as family history of migration, religious beliefs about the development and treatment of mental health problems, role of substance misuse without stereotyping cannabis use, experiences of racism, mistreatment within services, stigma associated with mental health problems and reluctance to seek help from mental health services. Significant emphasis was placed on the latter in terms of exploring how people may have been mistreated by services in the past and how this might perpetuate fear and mistrust in relation to help seeking. In relation to this, it was important for therapists to be particularly aware of confidentiality and cultural sanctions accompanying the risk of disclosure and negative perceptions of help-seeking from statutory services (as indicative of 'not coping'). In terms of the coping aspect of the intervention, it was recognized that cultural norms may inhibit 'talking your business' outside the family, so therapists needed to be mindful of this and help families acknowledge illness experiences and impact of symptoms. Relapse prevention (specifically, identification and management of early warning signs) and crisis planning were also conceptualized as being particularly important to promote more positive and less coercive routes to accessing to care.

Throughout therapy, attention was also drawn to the value placed upon food within the family home and its significance for hospitality, hierarchies within the family with greater respect given to elder members of the family, and not necessarily pathologizing raised voices within family discussions. In terms of the components of therapy, psychoeducation was reconceptualized as shared learning to emphasize how the therapists would also be learning 
about the family members' beliefs. Greater emphasis was placed on the need to learn about how the mental health system worked, including who to contact in a crisis given an overreliance on calling the police within a crisis in this group, and uses of and rights in relation to involuntary detentions given the higher levels of involuntary detention within the group.

In all instances, therapists were trained in cultural competence prior to starting therapy and were encouraged to revisit this learning through regular supervison. The level of training that therapists received in cultural competency in the trial has implications for roll-out across the health services, as currently this is not an integral part of healthcare professional training (Govere and Govere, 2016). This is despite the fact that cultural competence training is an effective means of enabling healthcare professionals to provide culturally competent care, which increases ethnic minorities' satisfaction with services (Govere and Govere, 2016).

\section{Conclusions}

Here we have presented three cases to illustrate CaFI for people with psychosis from AfricanCaribbean communities. We selected these cases in particular as they represent the range of service user and family member or FSM compositions that we experienced throughout the trial, including traditional family unit (mother and son dyad), service user and FSM from the community ('recruited FSM') and service users and mental health worker ('nominated FSM'). As illustrated above, in all instances therapists were able to focus on all components of the intervention, including assessment and engagement, shared learning, communication, coping skills and endings, suggesting that it was possible to adapt traditional FI in the absence of biological family members.

\section{Main points}

(1) This paper highlights that despite the challenges, it was possible to engage a diverse range of African-Caribbean people both with and without biological family members in FI for psychosis.

(2) Key themes arose in examples of therapy illustrated that were common in FI and CBT for psychosis for all ethnic groups, such as the importance of trauma and mental health services reinforcing past experiences of abuse.

(3) Having another person involved in therapy may enhance the therapeutic process and help maximize gains and in the absence of biological family members, mental health workers or volunteers from the community may take on this role.

(4) However, the involvement of non-family members raises questions about whether we are still delivering a family intervention or individual CBT.

\section{Acknowledgements}

We would like to thank all service users and their families who gave consent for details of their therapy to be included in the paper.

\section{Conflicts of interest}

The authors have no conflicts of interest with respect to this publication. 


\section{Ethical statement}

We abided by the Ethical Principles of Psychologists and Code of Conduct as set out by the APA. The study was approved by North West Greater Manchester East National Research Ethics Service (NRES) Ethics Committee (13/NW/0571).

\section{Financial support}

This work was supported by the National Institute of Health Research (grant number: $12 / 5001 / 62)$.

\section{Further suggested reading}

Barrowclough C, Tarrier N (1992). Families of Schizophrenic Patients: Cognitive Behavioural Intervention. London: Chapman and Hall.

Bucci S, Berry K, Barrowclough C, Haddock G (2016). Family interventions in psychosis: a review of the evidence and barriers to implementation. Australian Psychologist 51, 62-68.

Keating F, Robertson D (2004). Fear, black people and mental illness: a vicious circle? Health and Social Care in the Community 12, 439-447.

\section{References}

Barrowclough C, Tarrier N (1992). Families of Schizophrenic Patients: Cognitive Behavioural Intervention. London: Chapman and Hall.

Bebbington P, Jonas S, Kuipers E, King M, Cooper C, Brugha T et al. (2011). Childhood sexual abuse and psychosis: data from a cross-sectional psychiatric survey in England. The British Journal of Psychiatry 199, 29-37.

Berry K, Barrowclough C, Haddock G (2011). The role of expressed emotion in relationships between psychiatric staff and people with a diagnosis of psychosis: a review of the literature. Schizophrenia Bulletin 37, 958-972.

Berry K, Ford S, Jellicoe-Jones L, Haddock G (2013). PTSD symptoms associated with the experiences of psychosis and hospitalisation: a review of the literature. Clinical Psychology Review 33, 526-538.

Berry K, Haddock G (2008). The implementation of the NICE guidelines for schizophrenia: Barriers to the implementation of psychological interventions and recommendations for the future. Psychology and Psychotherapy: Theory, Research and Practice 81, 419-436.

Berry K, Wearden A, Barrowclough C (2007). Adult attachment styles and psychosis: an investigation of associations between general attachment styles and attachment relationships with specific others. Social Psychiatry and Psychiatric Epidemiology 42, 972-976.

Bucci S, Berry K, Barrowclough C, Haddock G (2016). Family interventions in psychosis: a review of the evidence and barriers to implementation. Australian Psychologist 51, 62-68.

Degnan A, Berry K, James S, Edge D (2018). Development, validation and cultural-adaptation of the knowledhe about psychosis questionnaire for African-Caribbean people in the UK. Psychiatry Research 263, 199-206.

Edge D, Degnan A, Cotterill S, Berry K, Baker J, Drake R, Abel K (2018). Culturally adapted Family Intervention $(\mathrm{CaFI})$ for African-Caribbean people diagnosed with schizophrenia and their families: a mixed methods feasibility study of development, implementation and acceptability. Health Services and Delivery Research 6, 1-348. 
Edge D, Degnan A, Cotterill S, Berry K, Drake R, Baker J et al. (2016). Culturally-adapted Family Intervention (CaFI) for African-Caribbeans diagnosed with schizophrenia and their families: a feasibility study protocol of implementation and acceptability. Pilot and Feasibility Studies 2, 39.

Fowler D, Garety P, Kuipers E (1995). Cognitive Behaviour Therapy for Psychosis: Theory and Practice. Chichester: Wiley.

Govere L, Govere EM (2016). How effective is cultural competence training of healthcase providers on improving patient satisfaction of minority groups? A systematic review of literature. Worldviews on Evidence-Based Nursing 13, 402-410.

Haddock G, Eisner E, Boone C, Davies G, Coogan C, Barrowclough C (2014). An investigation of the implementation of NICE-recommended CBT interventions for people with schizophrenia. Journal of Mental Health 23, 162-165.

Hagen R (2010). CBT for Psychosis: A Symptom-based Approach. London: Routledge.

Keating F, Robertson D (2004). Fear, black people and mental illness: a vicious circle? Health and Social Care in the Community 12, 439-447.

Lobban F, Postlethwaite A, Glentworth D, Pinfold V, Wainwright L, Dunn G (2013). A systematic review of randomised controlled trials of interventions reporting outcomes for relatives of people with psychosis. Clinical Psychology Review 33, 372-382.

Morgan C, Mallett R, Hutchinson G, Leff J (2004). Negative pathways to psychiatric care and ethnicity: the bridge between social science and psychiatry. Social Science and Medicine 58, 739752.

Morgan C, Fearon P, Lappin JM, Heslin M, Donoghue K, Lomas B et al. (2017). Ethnicity and long-term course and outcome of psychotic disorders in a UK sample: the AESOP-10 study. British Journal of Psychiatry 211, 88-94.

Morgan C, Kirkbride JB, Leff J, Craig T, Hutchinson G, McKenzie K, Jones P (2007). Parental separation, loss and psychosis in different ethnic groups: a case-control study. Psychological Medicine 37, 495-504.

National Institute for Health and Care Excellence (NICE) (2014). Psychosis and Schizophrenia in Adults: Treatment and Management. London: Department of Health.

Pharoah F, Mari J, Rathbone J, Wong W (2010). Family intervention for schizophrenia. Cochrane Database of Systematic Reviews, issue 12.

Qassem T, Bebbington P, Spiers N, McManus S, Jenkins R, Dein S (2015). Prevalence of psychosis in black ethnic minorities in Britain: analysis based on three national surveys. Social Psychiatry and Psychiatric Epidemiology 50, 1057-1064.

Rathod S, Phiri P, Harris S, Underwood C, Thagadur M, Padmanabi U, Kingdon D (2013). Cognitive behaviour therapy for psychosis can be adapted for minority ethnic groups: a randomised controlled trial. Schizophrenia Research 143, 319-326.

Schizophrenia Commission (2012). An abandoned illness: a report from the Schizophrenia Commission. London: Rethink Mental Illness.

Tortelli A, Errazuriz A, Croudace T, Morgan C, Murray RM, Jones PB, Szoke A, Kirkbride JB (2015). Scizophrenia and other psychotic disorders in Caribbean-born migrants and their descendants in England: systematic review and meta-analysis of incidence rates. Social Psychiatry and Psychiatric Epidemiology 50, 1039-1055.

Varese F, Smeets F, Drukker M, Lieverse R, Lataster T, Viechtbauer W et al. (2012). Childhood adversities increase the risk of psychosis: a meta-analysis of patient-control, prospective- and crosssectional cohort studies. Schizophrenia Bulletin 38, 661-671. 


\section{Learning objectives}

(1) To learn about specific challenges that might arise when trying to engage people from African-Caribbean backgrounds in family work for psychosis.

(2) To raise awareness of the possibility to delivering elements of a cognitive behavioural family intervention for psychosis when biological family members are not able or willing to engage.

(3) To raise awareness of the potential benefits of cultural competency training across the health service. 